\title{
Relación entre los factores que determinan los síntomas depresivos y los hábitos alimentarios en adultos mayores de México
}

\author{
José Alberto Ávila-Funes, ${ }^{1}$ Marie-Pierre Garant ${ }^{2}$ y Sara Aguilar-Navarro ${ }^{3}$
}

Forma de citar

Ávila-Funes JA, Garant MP, Aguilar-Navarro S. Relación entre los factores que determinan los síntomas depresivos y los hábitos alimentarios en adultos mayores de México. Rev Panam Salud Publica. 2006:19(5):321-30.

RESUMEN Objetivo. Determinar los factores comunes asociados tanto con los hábitos alimentarios de los adultos mayores que viven en la comunidad como con los sintomas depresivos.

Métodos. Se efectuó un análisis secundario de los datos provenientes de México, D.F., del estudio multicéntrico Salud, Bienestar y Envejecimiento (SABE) realizado entre 1999 y 2000. Se estudiaron las siguientes variables: presencia o ausencia de sintomas depresivos, según la escala de depresión geriátrica de Yesavage (EDG); el estado cognoscitivo, según la puntuación obtenida en una versión modificada del examen minimo del estado mental de Folstein (EMEM abreviado); y la funcionalidad, mediante el indice de actividades básicas de la vida diaria de Katz. A las personas que obtuvieron una suma total de 12 puntos o menos en el EMEM abreviado se les aplicó el cuestionario sobre actividades funcionales o de Pfeffer, para evaluar su capacidad de realizar las actividades cotidianas. Mediante autoinforme se obtuvo información acerca de las características materiales, físicas, psicológicas y sociales relacionadas con la compra, la preparación y el consumo de los alimentos y del estado de la salud bucal en los últimos 12 meses. Con las variables asociadas significativamente con el resultado de la EDG se realizó un análisis de regresión multifactorial, se elaboraron diversos modelos y se utilizaron las variables asociadas significativamente en un análisis de regresión lineal multifactorial por pasos para conocer el conjunto que mejor explicaba el resultado obtenido con la EDG.

Resultados. La edad promedio de los participantes fue de $64,4 \pm 8,6$ años y la prevalencia de sintomas depresivos fue de 66\%. El puntaje obtenido en la EDG mostró una asociación con la hipertensión arterial ( $\mathrm{P}<0,01)$, pero no con la presencia autoinformada de diabetes, neoplasias, enfermedad cerebrovascular, enfermedad pulmonar o enfermedad cardíaca. Sin embargo, el uso de prótesis dental $(\mathrm{P}<0,01)$, la incontinencia urinaria $(\mathrm{P}<0,01)$ y las caídas $(\mathrm{P}<0,01)$ se asociaron significativamente con los resultados de la EDG. El consumo de productos lácteos, carne, pescado y aves, y el de frutas y verduras fue significativamente menor en el grupo que presentaba síntomas depresivos. Hubo una correlación inversa entre el puntaje obtenido en la EDG por un lado y el número de comidas completas al día $(\mathrm{P}<0,01)$ y la cantidad de líquido ingerido $(\mathrm{P}<0,01)$ por el otro. Los factores determinantes que más estrecha asociación mostraron con esos resultados fueron, además de la hipertensión arterial, la presencia de alteraciones cognoscitivas $(\mathrm{P}<0,01)$, dificultades para realizar las actividades de la vida diaria básicas $(\mathrm{P}=0,03)$ e instrumentales $(\mathrm{P}<0,01)$, la poca movilidad $(\mathrm{P}<0,01)$, dificultad para usar el teléfono $(\mathrm{P}<0,01)$ y la autopercepción de tener mala memoria $(\mathrm{P}<0,01)$, de no

1 Centre de recherche sur le vieillissement, Institut universitaire de gériatrie de Sherbrooke, Université de Sherbrooke, Québec, Canadá. La correspondencia se debe enviar a J. Alberto Ávila-Funes, Centre de recherche sur le vieillissement, 1036,
Belvédère sud, Sherbrooke, Québec, Canada. J1H 4C4. Correo electrónico: avilaf1@hotmail.com

2 Centre de recherche clinique, Centre hospitalier universitaire de Sherbrooke, Québec, Canadá.
3 Clínica de Geriatría, Instituto Nacional de Ciencias Médicas y Nutrición Salvador Zubirán, México, D.F., México. 
tener suficientes recursos para vivir $(\mathrm{P}<0,01)$ y de tener mala salud bucal $(\mathrm{P}<0,01)$. Estas variables explicaron $31 \%$ de las variaciones observadas en los valores de $\mathrm{R}^{2}$ correspondientes a las variables de la EDG incorporadas al modelo explicativo final.

Conclusión. Varios factores determinantes de los síntomas depresivos y del resultado obtenido con la EDG son similares a los factores que determinan la aparición de trastornos en los hábitos alimentarios de los adultos mayores. La posibilidad de que existan mecanismos etiológicos comunes apunta a que se deben diseñar intervenciones dirigidas a prevenir la aparición y las consecuencias negativas de ambos trastornos. Estos resultados confirman la necesidad de tomar en cuenta los aspectos alimentarios, entre otros, durante el estudio de la depresión en los adultos mayores.

Palabras clave Depresión, desnutrición, anciano, alimentación, México.

La proporción de adultos mayores en la población mundial aumenta cada año. En México, por ejemplo, los ancianos representaban el $7,1 \%$ de la población (6,9 millones de personas) en el año 2000 y se prevé que su número ascienda a 32,4 millones en 2050, cuando uno de cada cuatro mexicanos tendrá más de 65 años (1). Conforme avanza la edad, algunas capacidades físicas se deterioran y aumenta la frecuencia de trastornos de salud. La mala nutrición es muy frecuente en los adultos mayores, debido en parte a los diversos cambios fisiológicos que ocurren durante el envejecimiento, muchos de los cuales llevan a reducir el consumo de alimentos (2). Sin embargo, una alimentación adecuada es fundamental para mantener la salud y la autonomía (3).

El equilibrio entre una buena alimentación y una adecuada capacidad funcional es frágil y, cuando se altera, la desnutrición puede provocar discapacidad (4) o el síndrome geriátrico que se conoce por "fragilidad" (5). La fragilidad es un trastorno que reduce la autonomía de los ancianos y dificulta su resistencia a las agresiones del medio ambiente o a las lesiones o enfermedades agudas (6). La pérdida del equilibrio de nitrógeno, propiciado por una ingesta reducida de alimentos, provoca la aparición o progresión de la pérdida de tejido muscular asociada con el envejecimiento, proceso conocido por sarcopenia (7). Este es un elemento clave de la fragilidad y está claramente asociado con la pérdida de la fuerza y de la autonomía $(8,9)$.
Los hábitos alimentarios de los adultos mayores son más heterogéneos que los del resto de la población y numerosos factores —su estado físico, los medios materiales de que disponen (como contar con utensilios de cocina o tener una prótesis dental), así como otros elementos psicológicos y socialespueden influir en su estado nutricional. Estos factores interactúan con los que determinan la cantidad y calidad de los alimentos que se compran, su preparación y su consumo. El desequilibrio en uno o varios de esos factores puede llevar al adulto mayor a padecer de desnutrición (10). Si bien la desnutrición no es un trastorno inherente del proceso de envejecimiento, cuando las condiciones de salud o ambientales son inapropiadas, el adulto mayor llega rápidamente a un estado de desnutrición que es difícil de revertir.

Se ha estimado que la prevalencia de desnutrición es de alrededor de $15 \%$ en los ancianos que viven en la comunidad (3). En América Latina - región caracterizada por grandes contrastes socioeconómicos-, los valores de prevalencia varían entre 4,6\% y $18 \%$, aunque en poblaciones más vulnerables, como la de los ancianos hospitalizados, puede llegar hasta $60 \%$ (11). La desnutrición está asociada con una elevada tasa de morbilidad (12), estancias hospitalarias prolongadas (13) con frecuentes readmisiones (14) y con una mayor mortalidad (15). Los factores que favorecen la desnutrición en el anciano son múltiples y muy disímiles; figuran entre ellos los trastornos de la deglución, la xerostomía o las enfermedades crónicas. También las enfermedades mentales y los trastornos de índole social pueden conducir a que su alimentación sea insuficiente.

La depresión es una enfermedad crónica frecuente en los adultos mayores y ocasiona muchos y diversos daños. Se estima que la prevalencia de síntomas depresivos entre los ancianos que viven en la comunidad en algunos países industrializados es de $10 \%$ a $27 \%$ (16-18). Diversas investigaciones demuestran que la depresión mayor - aunque también los síntomas depresivos- es un importante factor de riesgo de discapacidad, hospitalización y reclusión en instituciones y contribuye a elevar la morbilidad y la mortalidad (19-21), por lo que en general sus efectos son similares o incluso peores que los de otras enfermedades crónicas.

Al parecer, la sarcopenia, la disminución de la fuerza y la disminución de la capacidad física obedecen a mecanismos etiológicos similares a los de la desnutrición y la depresión, entre ellos algunos procesos inflamatorios y los efectos de algunas citocinas $(7,22$, 23). Además, la depresión también puede reducir los movimientos musculares voluntarios (24) y ello puede menoscabar el restablecimiento de la salud al dificultar que las personas busquen la ayuda necesaria para mejorar algunas limitaciones funcionales, mantener una alimentación adecuada o participar en actividades que mejoren su salud (19). Aunque se reconoce la relación existente entre la depresión y la desnutrición, no se conocen suficientemente bien los mecanismos etio- 
lógicos que estas enfermedades pueden tener en común (25).

El objetivo principal de este trabajo fue determinar los factores comunes asociados tanto con los hábitos alimentarios de los adultos mayores que viven en la comunidad como con los síntomas depresivos.

\section{MATERIALES Y MÉTODOS}

El presente estudio es un análisis secundario de los datos provenientes de la sección acerca de México, D.F., del estudio multicéntrico Salud, Bienestar y Envejecimiento (SABE). En ese estudio transversal se investigaron las condiciones de salud de los adultos mayores en ciudades de siete países de América Latina y el Caribe: Bridgetown, Barbados; Buenos Aires, Argentina; Ciudad de La Habana, Cuba; México, D.F., México; Montevideo, Uruguay; Santiago, Chile; y São Paulo, Brasil entre 1999 y 2000. Los detalles del protocolo se publicaron con anterioridad y están disponibles en Internet (26). El objetivo principal del proyecto SABE fue describir las condiciones de salud de los adultos mayores y su relación con las enfermedades agudas y crónicas, la discapacidad y el deterioro físico y mental. En esa investigación también se evaluaron el grado de acceso y uso de los servicios de salud y se compararon el apoyo familiar, la asistencia pública, el acceso a los servicios y los comportamientos saludables en las ciudades estudiadas. Además de las variables sociodemográficas habituales, la encuesta recabó información sobre el estado cognoscitivo, la situación de salud, el estado funcional, los hábitos alimentarios y el uso de y acceso a los servicios de salud entre adultos mayores en las ciudades estudiadas.

Se seleccionaron aleatoriamente personas mayores de 60 años y una muestra adicional de mujeres mayores de 50 años que vivían en el área metropolitana de México, D.F. Para ello se siguió un muestreo en tres etapas por conglomerados, con estratificación de los niveles más altos de agregación. Los participantes se identificaron a partir de la Encuesta de Hogares del Instituto Na- cional de Estadística, Geografía e Informática. La tasa de respuesta en México, D.F., fue de 83,7\%. En 5,9\% de las 1876 entrevistas logradas las respuestas las dieron representantes de la persona encuestada (su cónyuge o un pariente que viviera con ella). Toda la información recolectada, excepto las medidas antropométricas (peso, talla y circunferencia de cintura y cadera), la tensión arterial y la glucosa capilar, se obtuvieron a partir de autoinformes o de las respuestas de los representantes. Según el Censo de Población y Vivienda del año 2000, 632355 (7,3\%) habitantes de México, D.F., eran adultos mayores de más de 60 años de edad (1).

\section{Variables e instrumentos de medición}

Además de las características sociodemográficas necesarias para describir la muestra, se estudiaron las siguientes variables:

Síntomas depresivos. Los síntomas depresivos se evaluaron mediante la escala de depresión geriátrica de Yesavage (EDG) en su versión de 15 enunciados (27). Esta escala fue creada específicamente para adultos mayores (28) y se basa en un cuestionario que evalúa los síntomas específicos de la depresión geriátrica. El encuestado debía responder "sí" o "no" a cada pregunta sobre su estado de ánimo en las últimas dos semanas. El punto de corte para considerar que había síntomas depresivos era la respuesta afirmativa en al menos 6 de las 15 preguntas ( $\geq 6$ puntos) (27). Al igual que en su versión original de 30 preguntas, la versión abreviada utilizada en el estudio SABE tiene una validez y confiabilidad comprobadas, tanto en inglés como en español (29). Para el análisis no se tuvieron en cuenta las entrevistas en las que contestaron los representantes o en las que faltaban dos respuestas o más.

Hábitos alimentarios. Para identificar los elementos que reflejaban buenos hábitos de alimentación se utilizó el modelo teórico propuesto por Payette y col. (10). Este modelo se creó partiendo de información sobre adultos mayores que viven en la comunidad y explora las características materiales, físicas, psicológicas y sociales relacionadas con la compra, la preparación y el consumo de los alimentos. El modelo destaca, por ejemplo, la importancia de tener una movilidad adecuada (característica física) para salir a abastecerse de alimentos o de tener una prótesis dental correctamente ajustada (característica material) para poder consumirlos. La mayor parte de las preguntas relacionadas con los hábitos alimentarios en la encuesta SABE están inspiradas en las preguntas de la evaluación mínima del estado nutricional (Mini-Nutritional Assessment) (30). No obstante, algunas preguntas de este cuestionario fueron adaptadas para el estudio SABE y se adoptaron valores de referencia distintos de los recomendados durante la aplicación de la evaluación original. Las variables relacionadas con el gasto en alimentos, las instalaciones y los utensilios de cocina y el consumo de comidas comunitarias, contempladas en el modelo teórico de los hábitos alimentarios, no se incorporaron a la encuesta SABE.

Salud bucal. El índice de valoración de la salud bucal geriátrica (Geriatric Oral Health Assessment Index) se utilizó para cuantificar la necesidad de atención odontológica del adulto mayor mediante el autoinforme de su estado de salud bucal en los últimos 12 meses. La puntuación máxima era de 60 y una respuesta negativa en 3 de los 12 enunciados de la encuesta ( $<45$ puntos) significaba que la salud bucal no era satisfactoria (31).

Función mental. En la encuesta SABE se incluyó una versión modificada del examen mínimo del estado mental (Mini Mental State Examination, EMEM) de Folstein, Folstein y McHugh (32), denominado aquí EMEM abreviado. Esta modificación, desarrollada por Icaza y Albala, contiene las preguntas del EMEM que mejor reflejaron el deterioro cognoscitivo y posee una sensibilidad entre $82 \%$ y $94 \%$ y una especificidad entre $94 \%$ y $99 \%$ (33). La puntuación 
máxima es de 19 y se considera que un total por debajo de 13 indica que la persona presenta deterioro cognoscitivo.

Funcionalidad. Se utilizó el índice de Katz o de actividades básicas de la vida diaria (34) para evaluar la capacidad de realizar sin ayuda seis funciones básicas: bañarse, vestirse, usar el sanitario, trasladarse dentro del hogar, controlar los esfínteres y alimentarse. Se considera que este índice es un método objetivo para clasificar a grupos heterogéneos de personas con enfermedades crónicas y discapacidades, así como para describir sus necesidades de salud y evolución. Con un puntaje óptimo de 6 puntos (respuesta positiva a las 6 preguntas), se consideró que la pérdida de al menos una función reflejaba cierto grado de dependencia.

A las personas que obtuvieron una suma total de 12 puntos o menos en el EMEM abreviado se les aplicó el cuestionario sobre actividades funcionales (Functional Activities Questionnaire), conocido también como cuestionario de Pfeffer (35), para evaluar su capacidad de realizar las actividades cotidianas. Las respuestas a esta encuesta las dieron informantes o cuidadores que estuvieran acompañando a la persona con probable deterioro cognoscitivo. Aunque no evalúa la función mental, el cuestionario de Pfeffer detecta las actividades que pueden estar afectadas por problemas de la cognición - como cocinar, pagar las facturas o ir de compras- o que pueden reflejar un deterioro cognoscitivo. En el estudio SABE, este cuestionario consistió en 11 preguntas, calificadas entre 0 (normal) y 3 (completamente dependiente). Un total $\geq 6$ puntos indicaba la presencia de una discapacidad importante.

Hábitos de vida. Se exploraron el consumo semanal de tabaco y el consumo de alcohol como variables categóricas ordinales.

\section{Análisis estadístico}

Se calcularon la media y la desviación estándar de las variables continuas y la frecuencia y el porcentaje de las variables categóricas (36). Se identificaron mediante análisis de correlación los factores determinantes de los hábitos alimentarios que mejor se correlacionaron con el resultado de la EDG (variable dependiente). El resultado de la EDG también se tomó como variable dicotómica (presencia o ausencia de síntomas depresivos). Para demostrar la relación significativa entre las variables se utilizó el coeficiente de correlación (de Pearson o de Spearman), la prueba de la $t$ de Student o la de la ji al cuadrado, según el tipo de variable. Se emplearon el análisis bifactorial y la prueba de la ji al cuadrado para explorar la asociación entre el sexo de la persona y la presencia de los síntomas depresivos. Con las variables asociadas significativamente con el resultado de la EDG se realizó un análisis de regresión multifacto-rial. Según el grupo de las variables —sociodemográficas o de actividades de la vida diaria - se elaboraron diversos modelos y se utilizaron las variables asociadas significativamente en un análisis de regresión lineal multifactorial por pasos para conocer el conjunto que mejor explicaba el resultado obtenido con la EDG.

Posteriormente se verificó la validez de la regresión lineal mediante el análisis de valores residuales y se realizó un análisis de regresión logística para saber si las variables identificadas por el método lineal se asociaban también de forma independiente con los síntomas depresivos. Se utilizó un nivel de significación estadística $P<0,05$. Para todos los análisis se utilizó el paquete estadístico SPSS versión 12.0 para Windows ${ }^{\circledR}$ (SPSS Inc., Chicago, IL).

\section{RESULTADOS}

De 1876 entrevistas realizadas, se eliminaron 111 del análisis de síntomas depresivos mediante la EDG porque los entrevistados no habían sido los propios participantes y 17 porque faltaban dos respuestas o más. En total, 1748 personas conformaron la muestra final; 465 (26,6\%) fueron hombres y $1283(73,4 \%)$ fueron mujeres. La edad promedio fue de $64,4 \pm 9,6$ años $y$ la media de la puntuación total en la EDG fue de 6,85 $\pm 2,17$. El resto de las características generales de la muestra se presentan en el cuadro 1. La correlación de la EDG con el índice de masa corporal no fue significativa $(P=0,10)$, pero sí lo fue con el índice cinturacadera $(r=0,07 ; P<0,01)$.

Las variables más importantes desde el punto de vista clínico que mostraron una asociación significativa con el resultado de la EDG en el análisis bifactorial se presentan en el cuadro 2. La correlación encontrada fue débil o moderada. La puntuación en la EDG estuvo asociada con la hipertensión arterial $(P<0,01)$, pero no con el consumo de medicamentos ni con la variación de peso corporal debido a la hipertensión arterial crónica. No se encontró relación entre la EDG y la presencia autoinformada de diabetes, neoplasias, enfermedad cerebrovascular, enfermedad pulmonar o enfermedad cardíaca. Sin embargo, el uso de una prótesis dental $(P<0,01)$, la incontinencia urinaria $(P<0,01)$ y las caídas $(P<0,01)$ se asociaron significativamente con el resultado de la EDG, al igual que otras variables dicotómicas relacionadas con la capacidad para realizar actividades de la vida diaria: dificultad para realizar actividades físicas rigurosas, permanecer sentado por más de dos horas o levantarse de una silla, subir al menos un piso, agacharse, extender los brazos, transportar un bulto, comprar los alimentos y preparar la comida, manejar el dinero, salir de la casa, tomar los medicamentos y realizar actividades manuales. También se asoció con no tener lo suficiente para vivir, según lo informado por el entrevistado.

En cuanto a los hábitos de vida, no se observó ninguna asociación significativa entre la presencia de síntomas depresivos y la intensidad del consumo de alcohol o con el consumo de tabaco, vivir solo o haber manifestado la necesidad de ayuda para preparar los alimentos.

Los cuadros 3 y 4 muestran la relación entre las variables más importantes desde el punto de vista clínico y los síntomas depresivos, según el sexo. En las categorías en que se observaron 
CUADRO 1. Características generales de la muestra estudiada ( $n=1784$ ). México, D.F., 1999-2000

\begin{tabular}{|c|c|c|}
\hline Variable & $\%$ & Media $\pm \mathrm{DE}^{\mathrm{a}}$ \\
\hline Edad (años) & $N A^{b}$ & $64,4 \pm 9,6$ \\
\hline Hombres & 26,6 & NA \\
\hline Mujeres & 73,4 & NA \\
\hline Sabe leer y escribir un recado & 83,6 & NA \\
\hline Mala autopercepción de memoria & 6,8 & NA \\
\hline Mala autopercepción de salud & 17,2 & NA \\
\hline Vivían solos & 9,7 & NA \\
\hline Percibían tener lo suficiente para vivir & 48,9 & NA \\
\hline Dolor articular & 17,4 & NA \\
\hline Se habían caído en los últimos 12 meses & 33,9 & NA \\
\hline Usaban dentadura postiza & 50,1 & NA \\
\hline Se consideraban bien nutridos & 76,9 & NA \\
\hline Hipertensión arterial & 41 & $132,9 \pm 18,8 \mathrm{~mm} \mathrm{Hg}^{\mathrm{c}}$ \\
\hline Diabetes mellitus & 19,8 & $132,9 \pm 60,2 \mathrm{mg} / \mathrm{dL}^{\mathrm{d}}$ \\
\hline Índice de masa corporal & NA & $28,6 \pm 5,0 \mathrm{~kg} / \mathrm{m}^{2}$ \\
\hline Índice cintura-cadera & NA & $0,90 \pm 0,7$ \\
\hline $\begin{array}{l}\text { Presenta síntomas depresivos según la escala } \\
\text { de depresión geriátrica }(\geq 6)\end{array}$ & 66,0 & $6,85 \pm 2,17$ \\
\hline $\begin{array}{l}\text { Presenta discapacidad funcional importante según } \\
\text { la escala de Pfeffer }(\geq 6)(n=217)\end{array}$ & 32,3 & $6,0 \pm 9,0$ \\
\hline $\begin{array}{l}\text { Presenta mala salud bucal según el índice } \\
\text { de valoración de la salud bucal geriátrica }\end{array}$ & & \\
\hline$(\leq 45)(n=1706)$ & 326,2 & $47,8 \pm 7$ \\
\hline Índice de Katz & NA & $5,4 \pm 1,0$ \\
\hline
\end{tabular}

a Desviación estándar.

b NA: no se aplica.

c Tensión arterial sistólica.

d Glucosa capilar.

CUADRO 2. Correlaciones entre la puntuación total de la escala de depresión geriátrica y algunas variables de importancia clínicaa. México, D.F., 1999-2000

\begin{tabular}{|c|c|c|}
\hline Variable & $\begin{array}{c}\text { Coeficiente de } \\
\text { correlación }^{b}\end{array}$ & $P$ \\
\hline Índice de valoración de la salud bucal geriátrica & $r=-0,31$ & $<0,01$ \\
\hline Preocupación por los dientes ${ }^{a}$ & $r_{\mathrm{s}}=-0,25$ & $<0,01$ \\
\hline Problemas para masticara & $r_{s}=-0,24$ & $<0,01$ \\
\hline Índice de Katz & $r=-0,30$ & $<0,01$ \\
\hline Autopercepción de saluda & $r_{s}=0,30$ & $<0,01$ \\
\hline Puntaje en el examen mínimo del estado mental abreviado & $r=-0,27$ & $<0,01$ \\
\hline Autopercepción de memoria ${ }^{a}$ & $r_{\mathrm{s}}=0,27$ & $<0,01$ \\
\hline Limitaciones por dolor articular ${ }^{a}$ & $r_{\mathrm{s}}=-0,20$ & $<0,01$ \\
\hline Escala de Pfeffer & $r=0,16$ & $<0,05$ \\
\hline Autopercepción de comer menos ${ }^{a}$ & $r_{s}=-0,16$ & $<0,01$ \\
\hline Autopercepción de la visióna & $r_{\mathrm{s}}=0,15$ & $<0,01$ \\
\hline Autopercepción de la pérdida de peso ${ }^{a}$ & $r_{\mathrm{s}}=-0,13$ & $<0,01$ \\
\hline
\end{tabular}

diferencias significativas, el análisis bifactorial demostró que el sexo del entrevistado no se asoció con la presencia o ausencia de síntomas depresivos; sí se asoció con saber leer y escribir
( $P=0,05$ en hombres y $P<0,01$ en mujeres), con tener hipertensión arterial (solo en mujeres, $P<0,01$ ) y con el consumo de lácteos (solo en mujeres, $P<0,01)$.
El consumo de productos lácteos, carne, pescado y aves, y el de frutas y verduras fue significativamente menor en el grupo que presentaba síntomas depresivos (cuadro 4). También hubo una correlación inversa entre el puntaje obtenido en la EDG por un lado y el número de comidas completas al día $(P<0,01)$ y la cantidad de líquido ingerido $(P<0,01)$ por el otro.

En el cuadro 5 se presentan los parámetros del modelo de regresión lineal con múltiples variables de los factores que tuvieron mayor valor pronóstico en relación con la puntuación total obtenida en la EDG. Este modelo general fue significativo $(F=43,02 ; P<0,01) \mathrm{y}$, en su conjunto, logró explicar $31 \%$ de las variaciones observadas en los resultados de la EDG. Finalmente, según el análisis de regresión logística, 9 de las 12 variables incorporadas al modelo lineal (excepto "dificultad para agacharse", "dificultad para manejar el dinero" y "dificultad para hablar debido a problemas dentales") conformaron la ecuación de predicción y esta se asoció significativamente con la presencia de síntomas depresivos $(P<0,01)$, pero su valor pronóstico en un nuevo modelo lineal fue menor $\left(R^{2}=22 \%\right)$.

\section{DISCUSIÓN}

Los resultados de este estudio demuestran que varios de los factores que determinan el resultado en la EDG - e incluso la presencia de síntomas depresivos - son similares a los que determinan los hábitos alimentarios de los adultos mayores. Algunas características, ya sean materiales, físicas o psicológicas, incorporadas al modelo explicativo final están parcialmente relacionadas con la compra, preparación y consumo de los alimentos. A pesar de que un gran número de variables mostraron asociaciones significativas con el resultado de la EDG o con la presencia de síntomas depresivos, se comprobó que algunas de ellas tienen un mayor valor pronóstico.

La depresión en el anciano está asociada con varios síntomas, entre ellos la pérdida de peso (37), que constituye un signo de alerta de suma impor- 
CUADRO 3. Relación entre las principales variables clínicas continuas y la presencia de síntomas depresivos, según el sexo. México, D.F., 1999-2000

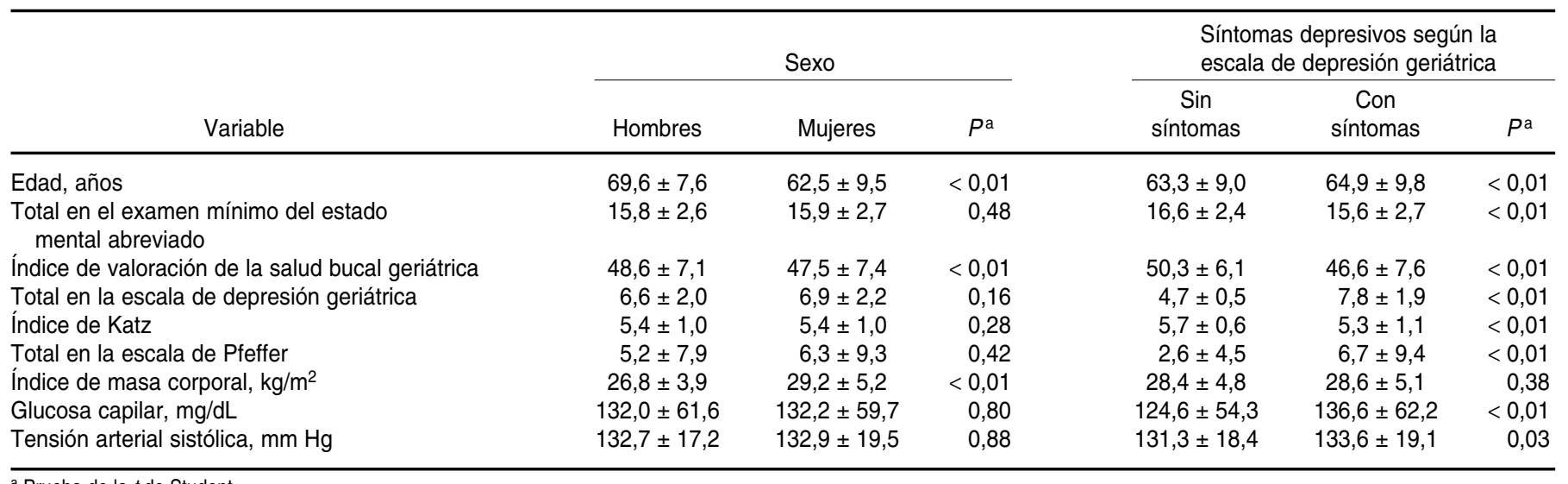

a Prueba de la $t$ de Student.

CUADRO 4. Relación entre las principales variables clínicas categóricas y la presencia de síntomas depresivos, según el sexo. México, D.F., 1999-2000

\begin{tabular}{|c|c|c|c|c|c|c|}
\hline \multirow[b]{2}{*}{ Variable } & \multicolumn{3}{|c|}{ Sexo } & \multicolumn{3}{|c|}{$\begin{array}{l}\text { Síntomas depresivos según la } \\
\text { escala de depresión geriátrica }\end{array}$} \\
\hline & $\begin{array}{l}\text { Hombres } \\
\%\end{array}$ & $\begin{array}{l}\text { Mujeres } \\
\%\end{array}$ & $P^{a}$ & $\begin{array}{c}\text { Sin } \\
\text { síntomas } \\
\%\end{array}$ & $\begin{array}{c}\text { Con } \\
\text { síntomas } \\
\%\end{array}$ & $P^{a}$ \\
\hline Sabe leer y escribir & 87 & 82 & $<0,01$ & 89 & 80 & $<0,05^{b}$ \\
\hline Vive solo & 9,5 & 10 & 0,83 & 9,2 & 9,8 & 0,67 \\
\hline Hipertensión arterial & 35 & 43 & $<0,01$ & 33 & 45 & $<0,01^{\mathrm{c}}$ \\
\hline Diabetes mellitus & 23 & 19 & 0,06 & 14,3 & 22,5 & $<0,01$ \\
\hline Cáncer & 1,2 & 2,8 & 0,18 & 3 & 2 & 0,42 \\
\hline Cardiopatía isquémica & 9,8 & 8,3 & 0,42 & 6,4 & 9,8 & 0,04 \\
\hline Enfermedad vascular cerebral & 5,8 & 3,6 & 0,10 & 2,5 & 5 & 0,03 \\
\hline Neumopatía & 7,8 & 9,3 & 0,34 & 5,2 & 10,5 & $<0,01$ \\
\hline Consumo de lácteos al menos una vez al día & 86,8 & 97,7 & 0,05 & 92,9 & 87,5 & $<0,01^{\mathrm{c}}$ \\
\hline Consumo de huevos y frijoles & 96,7 & 95 & 0,13 & 96,3 & 95,1 & 0,25 \\
\hline Consumo de carne, pescado o aves & 85,9 & 86,0 & 0,94 & 91,6 & 83,7 & $<0,01$ \\
\hline Consumo de frutas y verduras & 84,4 & 86,4 & 0,50 & 88,5 & 84,6 & 0,03 \\
\hline Fuma actualmente & 29,4 & 11,1 & $<0,01$ & 16,9 & 15,4 & 0,64 \\
\hline Nunca bebió alcohol & 65,5 & 87,2 & $<0,01$ & 82,2 & 81,3 & 0,92 \\
\hline
\end{tabular}

a Prueba de la ji al cuadrado.

${ }^{b}$ El sexo influyó en la diferencia: $P=0,05$ en hombres y $P<0,01$ en mujeres.

c Solo en mujeres.

tancia en los adultos mayores por su asociación con la desnutrición $(4,38)$. Los mecanismos que intervienen en esta asociación no se han estudiado a fondo. Es probable que la pérdida del apetito durante el envejecimiento conduzca a un equilibrio de nitrógeno negativo, a la aparición de sarcopenia y a la pérdida de peso, síntomas que están relacionados con la depresión y la fragilidad $(39,40)$.

Los factores independientes que tienen un valor pronóstico respecto del resultado de la EDG y de la presencia de los síntomas depresivos, identificados mediante el análisis de regresión, pueden tener elementos en común. Según algunos autores, ciertas enfermedades crónicas - específicamente la hipertensión arterial- están asociadas con la presencia de lesiones vasculares en áreas específicas del cerebro (41), las cuales pueden contribuir a la aparición de síntomas depresivos en la vejez. Estas lesiones pueden ocurrir en determinados circuitos cerebrales (del lóbulo frontal o el putamen, por ejemplo) que desempeñan un papel importante en la realización de tareas que exigen una función ejecutiva adecuada, como el control del dinero (42, 43). También se ha relacionado la depresión con los trastornos de la marcha ocasionados por lesiones en el sistema nervioso central (44).

En este estudio, $41 \%$ de los participantes informaron tener hipertensión arterial, por lo que hipotéticamente pudieron haber sufrido alguna lesión vas- 
CUADRO 5. Determinantes de los hábitos alimentarios del adulto mayor que mejor predijeron la puntuación total obtenida en la escala de depresión geriátricaa. México, D.F., 1999-2000

\begin{tabular}{lrrr}
\hline \multirow{2}{*}{\multicolumn{1}{c}{ Variable }} & \multicolumn{3}{c}{ Coeficientes } \\
\cline { 2 - 4 } & Beta & Error & \multicolumn{1}{c}{$P$} \\
\hline Examen mínimo del estado mental abreviado & $-0,143$ & 0,022 & $<0,01$ \\
Autopercepción de mala memoria & 0,274 & 0,067 & $<0,01$ \\
Autopercepción de comer menos & 0,777 & 0,125 & $<0,01$ \\
Índice de Katz & $-0,167$ & 0,075 & 0,02 \\
Dificultad para levantarse de una silla & 0,391 & 0,127 & $<0,01$ \\
Dificultades para agacharse & 0,303 & 0,126 & 0,01 \\
Dificultad para administrar el dinero & 1,315 & 0,447 & $<0,01$ \\
Dificultades para llamar por teléfono & 0,801 & 0,266 & $<0,01$ \\
Preocupación por los dientes & $-0,293$ & 0,053 & $<0,01$ \\
Dificultar al hablar por problemas dentales & $-0,171$ & 0,051 & $<0,01$ \\
No tener recursos suficientes para vivir & $-0,398$ & 0,111 & $<0,01$ \\
Tener hipertensión arterial & 0,352 & 0,110 & $<0,01$ \\
\hline
\end{tabular}

a Constante: 10,$636 ; R^{2}=0,306$.

cular en el sistema nervioso central que les provocara dificultades para procesar la información en ese nivel y que se manifestara con síntomas depresivos y problemas en el desempeño ejecutivo. La depresión y la dificultad para realizar actividades ejecutivas podrían repercutir en la autopercepción de la salud, la funcionalidad y la realización de las actividades que de ella dependen, como las actividades básicas e instrumentales de la vida diaria.

Se sabe desde hace muchos años que la presencia de síntomas depresivos se asocia con el deterioro de la función cognoscitiva (45) y algunos autores han demostrado que ambos trastornos constituyen factores de riesgo de demencia (46). En el presente estudio, $8,2 \%$ de los participantes presentaban deterioro cognoscitivo, según la puntuación total obtenida en el EMEM abreviado, y 32,3\% de ellos habían sufrido una pérdida parcial de la capacidad funcional, según su puntuación en la escala de Pfeffer. No obstante, este subgrupo fue semejante al resto de los participantes en los demás aspectos, por lo que el deterioro cognoscitivo no parece ser un factor pronóstico en relación con los síntomas depresivos.

La autopercepción de mala salud es otro factor determinante de los síntomas depresivos que influye en el aumento de la morbilidad y mortalidad (47). La percepción de tener mala memoria y mala salud tuvo una correla- ción positiva con los síntomas de depresión, aunque la segunda no formó parte del modelo explicativo final. La percepción errónea del estado de la memoria y de la salud y la mala interpretación de los sucesos habituales de la vida pueden favorecer la aparición de la depresión. Esto lo explica el modelo psicológico de distorsión cognoscitiva, según el cual las personas que peor perciben los sucesos son las que se deprimen y viceversa. Según ese modelo, la distorsión de la función cognoscitiva puede provocar que el adulto mayor se cree expectativas alejadas de la realidad o que generalice y reaccione de manera exagerada ante sucesos negativos (48).

Llama la atención que en la muestra estudiada, las personas con síntomas depresivos expresaron tener dificultades para salir de la casa o preparar los alimentos, pero al mismo tiempo negaron necesitar ayuda con dichas actividades. El origen "psicológico" de muchos casos de depresión puede explicar, al menos en parte, la percepción de muchos participantes con síntomas depresivos, detectados mediante las escalas empleadas en este estudio, de que su estado general no era satisfactorio.

Por otro lado, dos preguntas del índice de valoración de la salud bucal geriátrica ("estar nervioso o tener dificultades para hablar por confrontar problemas con los dientes") formaron parte del modelo explicativo final. A pesar de que la puntuación total obtenida en este índice arrojó valores significativos en los modelos de regresión preliminares $\left(R^{2}=0,105\right)$, no fue significativa en el modelo final. Estudios transversales han demostrado que la mala higiene bucal y la inferior calidad de vida asociada con una mala salud bucal son mucho más frecuentes en personas con depresión que en las que no están deprimidas $(49,50)$. Sin embargo, es posible que esta asociación sea bidireccional, ya que la mala salud bucal puede desencadenar o ser indicio de algún trastorno mental subyacente (como estrés, ansiedad o depresión) y la depresión puede, a su vez, favorecer los malos hábitos de salud y la negligencia en el cuidado personal.

Varias funciones relacionadas con la movilidad fueron factores pronóstico independientes en relación con los síntomas depresivos. Se ha informado que las limitaciones de la movilidad están asociadas con la presencia de síntomas depresivos ${ }^{4}$. Según Kiosses y col. (51), los ancianos con problemas cognoscitivos, particularmente en las funciones ejecutivas, tenían también dificultades para realizar las actividades instrumentales de la vida diaria - especialmente comprar y preparar los alimentos, tomar los medicamentos y administrar el dinero-, pero esa asociación fue más fuerte en las personas que también presentaban síntomas depresivos. Los cambios en el comportamiento debidos a las dificultades para realizar funciones ejecutivas y la depresión pueden traducirse en falta de interés o motivación, lo que puede explicar el aprovisionamiento insuficiente o la alimentación inadecuada.

La autopercepción de tener dificultades financieras, sobre todo para proveerse de alimentos, es particularmente importante en las personas con síntomas depresivos, lo que puede explicar que esta variable resultara un factor pronóstico independiente. En el estudio transversal de Klesges et al. (52), el

\footnotetext{
4 Hirvensalo M, Sakari-Rantala R, Leinonnen R, Lintunen T, Rasinaho M, Heikkinen E, et al. Depressed mood and mobility limitation in older people. [Presentación en póster]. 18th Congress of the International Association of Gerontology, Rio de Janeiro, 26-30 de junio de 2005.
} 
hecho de no tener suficientes recursos para adquirir comida se asoció con la depresión y la mala calidad de vida.

La omisión en el análisis de algunas variables del modelo teórico de los hábitos alimentarios (por no estar disponible en la base de datos de SABE correspondiente a México) probablemente sea la causa de que los modelos de regresión expliquen solamente $31 \%$ de las variaciones observadas en los valores de $R^{2}$ de las variables de la EDG incorporadas al modelo explicativo final. Sin embargo, algunas variables, tales como "dinero gastado en los alimentos", "disponibilidad de utensilios de cocina" o "compañía en el momento de las comidas", pueden inferirse a partir de otras variables, como "suficiencia económica para vivir" y "vivir solo", aunque no se correspondan totalmente. Otras variables que no están contempladas en el modelo teórico de Payette y col. (10) podrían quizá explicar mejor la presencia de síntomas de depresión en la población mexicana de más de 60 años.

Aunque se ha demostrado que los factores determinantes de los hábitos alimentarios identificados en este estudio están asociados con los síntomas depresivos de forma individual $(38,41$, $45,47,49,50-52)$, esta es la primera vez que se demuestra una posible interrelación entre los factores que determinan los hábitos alimentarios y los que determinan los síntomas depresivos.

Este estudio tiene varias limitaciones. Primero, su naturaleza transversal no permite establecer la dirección causal de las asociaciones encontradas, aunque algunos autores han encontrado una relación bidireccional entre la depresión y la discapacidad $(17,53)$. Segundo, la información relacionada con la salud y la funcionalidad se obtuvo mediante autoinformes, con las limitaciones que ello implica. En los grandes estudios epidemiológicos muchas veces no es posible (por restricciones logísticas o económicas) evaluar objetivamente la funcionalidad ni lograr que profesionales de la salud evalúen a los participantes para corroborar la presencia de enfermedades. Sin embargo, aunque estos resultados deben interpretarse cuidadosamente, ya que pueden contener varios errores, los autoinformes han demostrado suficiente validez y representatividad en el estudio de variables subjetivas, tales como la salud y la calidad de la vida $(54,55)$. Tercero, el modelo teórico de Payette y col. sobre los hábitos alimentarios de los adultos mayores se elaboró a partir de información obtenida en la población de los Estados Unidos y Canadá. Por consiguiente, las variables incorporadas a ese modelo no representan necesariamente los mismos mecanismos en la población latinoamericana. Finalmente, la encuesta SABE no fue diseñada específicamente para responder a los objetivos de este trabajo. Se deben realizar más estudios sobre los factores determinantes de los hábitos alimentarios en la población latinoamericana, ya que los aspectos sociales pueden

\section{REFERENCIAS}

1. Instituto Nacional de Estadística, Geografía e Informática. Estadísticas a propósito del día internacional de las personas de la tercera edad. [Sitio en Internet]. Hallado en: http:// $\mathrm{www}$.inegi.gob.mx/inegi/contenidos/ espanol/temp/prensa/contenidos/estadisticas/ personas.pdf. Acceso el 11 de septiembre de 2005

2. Morley JE, Thomas DR. Anorexia and aging: pathophysiology. Nutrition. 1999;15(6):499-503.

3. Fleming KC, Evans JM, Weber DC, Chutka DS. Practical functional assessment of elderly persons: a primary-care approach. Mayo Clin Proc. 1995;70(9):890-910.
4. Payette H. Stratégies de prévention de la malnutrition chez les personnes âgées en perte d'autonomie a domicile. Age Nutr. 1996;7(1): 6-9.

5. Morley JE. Decreased food intake with aging. J Gerontol A Biol Sci Med Sci. 2001;56(Sp2): 81-8.

6. Fried LP, Tangen CM, Walston J, Newman AB, Hirsch C, Gottdiener J, et al. Frailty in older adults: evidence for a phenotype. J Gerontol A Biol Sci Med Sci. 2001;56(3): M146-56.

7. Ferrucci L, Penninx BW, Volpato S, Harris TB, Bandeen-Roche K, Balfour J, et al. Change in muscle strength explains accelerated decline tener una importancia mayor debido a las particularidades del trato al adulto mayor en nuestra cultura.

A pesar de esas limitaciones, los resultados obtenidos indican que varios factores determinantes de los síntomas depresivos y del resultado en la EDG influyen también en los hábitos alimentarios de los adultos mayores. En teoría, los daños del sistema nervioso central, provocados por algunas enfermedades crónicas, como la hipertensión, pudieran provocar alteraciones cognoscitivas y del comportamiento que lleven a la aparición de síntomas depresivos, a comportamientos poco sanos y a diversas discapacidades. De esta manera, el control adecuado de la tensión arterial podría ayudar a prevenir discapacidades, muchas de ellas coadyuvadas por la depresión y la desnutrición.

Estos resultados confirman la necesidad de tomar en cuenta los aspectos alimentarios durante el estudio de la depresión en los adultos mayores. La posibilidad de que existan mecanismos etiológicos comunes apunta a la necesidad de diseñar intervenciones dirigidas a prevenir la aparición y las consecuencias negativas de ambos trastornos. Se deben realizar estudios longitudinales en poblaciones sin discapacidades, síntomas depresivos y riesgo de desnutrición para poder identificar con el paso del tiempo los factores materiales, físicos, psicológicos y sociales que favorecen la aparición de la depresión y de la desnutrición. of physical function in older women with high interleukin-6 serum levels. J Am Geriatr Soc. 2002;50(12):1947-54.

8. Roubenoff R, Hughes VA. Sarcopenia: current concepts. J Gerontol A Biol Sci Med Sci. 2000; 55(12):M716-24.

9. Fried LP, Hadley EC, Walston JD, Newman A, Guralnik JM, Studenski S, et al. From bedside to bench: research agenda for frailty. Sci Aging Knowl Environ. 2005;2005(31):pe24.

10. Payette H, Gray-Donald K, Cyr R, Boutier V. Predictors of dietary intake in a functionally dependent elderly population in the community. Am J Public Health. 1995;85(5):677-83. 
11. Correia MI, Campos AC. Prevalence of hospital malnutrition in Latin America: the multicenter ELAN study. Nutrition. 2003;19(10): 823-5.

12. Sullivan DH, Walls RC. Impact of nutritional status on morbidity in a population of geriatric rehabilitation patients. J Am Geriatr Soc. 1994;42(5):471-7.

13. Chima CS, Barco K, Dewitt ML, Maeda M, Teran JC, Mullen KD. Relationship of nutritional status to length of stay, hospital costs, and discharge status of patients hospitalized in the medicine service. J Am Diet Assoc. 1997;97(9):975-8.

14. Sullivan DH. Impact of nutritional status on health outcomes of nursing home residents. J Am Geriatr Soc. 1995;43(2):195-6.

15. Reilly JJ Jr, Hull SF, Albert N, Waller A, Bringardener S. Economic impact of malnutrition: a model system for hospitalized patients. J Parenter Enteral Nutr. 1988;12(4):371-6.

16. Ormel J, Koeter MW, van den Brink W, van de Willige G. Recognition, management, and course of anxiety and depression in general practice. Arch Gen Psychiatry. 1991;48(8): 700-6.

17. Penninx BW, Guralnik JM, Ferrucci L, Simonsick EM, Deeg DJ, Wallace RB. Depressive symptoms and physical decline in communitydwelling older persons. J Am Med Assoc. 1998;279(21):1720-6.

18. Andrade L, Caraveo-Anduaga JJ, Berglund P, Bijl RV, De Graaf R, Vollebergh W, et al. The epidemiology of major depressive episodes: results from the International Consortium of Psychiatric Epidemiology (ICPE) Surveys. Int J Methods Psychiatr Res. 2003;12(1):3-21.

19. Cronin-Stubbs D, de Leon CF, Beckett LA, Field TS, Glynn RJ, Evans DA. Six-year effect of depressive symptoms on the course of physical disability in community-living older adults. Arch Intern Med. 2000;160(20):3074-80.

20. Geerlings SW, Beekman AT, Deeg DJ, Twisk JW, Van Tilburg W. The longitudinal effect of depression on functional limitations and disability in older adults: an eight-wave prospective community-based study. Psychol Med. 2001;31(8):1361-71.

21. Penninx BW, Guralnik JM, Bandeen-Roche K, Kasper JD, Simonsick EM, Ferrucci L, et al. The protective effect of emotional vitality on adverse health outcomes in disabled older women. J Am Geriatr Soc. 2000;48(11):1359-66.

22. Katz IR. Depression and frailty: the need for multidisciplinary research. Am J Geriatr Psychiatry. 2004;12(1):1-6.

23. Payette H, Roubenoff R, Jacques PF, Dinarello CA, Wilson PW, Abad LW, et al. Insulin-like growth factor-1 and interleukin 6 predict sarcopenia in very old community-living men and women: the Framingham Heart Study. J Am Geriatr Soc. 2003;51(9):1237-43.

24. Allen GM, Hickie I, Gandevia SC, McKenzie DK. Impaired voluntary drive to breathe: a possible link between depression and unexplained ventilatory failure in asthmatic patients. Thorax. 1994;49(9):881-4.

25. Johnson J. Psychosocial correlates of nutritional risk in older adults. Can J Diet Prac Res. 2005;66(2):95-7.
26. Peláez M, Palloni A, Albala C, Alfonso JC, Ham-Chande R, Hennis A, et al. SABE-Survey on health, well-being, and aging in Latin America and the Caribbean, 2000. [Computer file]. ICPSR version. Washington, D.C.: Pan American Health Organization/World Health Organization [producers]; 2004. Ann Arbor, MI: Inter-University Consortium for Political and Social Research [distributor]; 2005. Hallado en: http://www.icpsr.umich.edu/pdf/ 03546/User_s_Manual.pdf. Acceso el 11 de septiembre de 2005.

27. Yesavage JA. Geriatric depression scale. Psychopharmacol Bull. 1988;24(4):709-11.

28. Brink TL, Yesavage JA, Lum O, Heersema PH, Adey M, Rose TL. Screening tests for geriatric depression. Clin Gerontol. 1982;1(1):37-43.

29. Martínez de la Iglesia J, Onis Vilches MC, Dueñas Herrero R, Aguado Taberne C, Albert Colomer C, Arias Blanco MC. Abreviar lo breve. Aproximación a versiones ultracortas del cuestionario de Yesavage para el cribado de la depresión. Aten Prim. 2005;35(1):14-21.

30. Guigoz Y, Vellas B. Mini-nutritional assessment: a practical assessment tool for grading the nutritional state of elderly patients. Facts Rese Gerontol. 1994;(Suppl2):15-59.

31. Atchinson KA, Dolan TA. Development on the geriatric oral health assessment index. J Dent Educ. 1990;54(11):680-6.

32. Folstein MF, Folstein SE, McHugh PR. Minimental state. A practical method for grading the cognitive state of patients for the clinician. J Psychiatr Res. 1975;12(3):189-98.

33. Icaza MG, Albala C. Mini-Mental State Examination (MMSE) del estudio de la demencia en Chile: análisis estadístico. Serie Investigaciones en Salud Pública. [Sitio en Internet]. Hallado en: http://www.paho.org/spanish/ $\mathrm{hdp} / \mathrm{hdr} /$ serie07composite.pdf. Acceso el 11 de septiembre de 2005.

34. Katz S, Akpom CA. Index of ADL. Med Care 1976;14(Suppl5):116-8.

35. Pfeffer RI, Kurosaki TT, Harrah CH Jr, Chance JM, Filos S. Measurement of functional activities in older adults in the community. J Gerontol. 1982;37(3):323-9.

36. Altman DG, Bland JM. Presentation of numerical data. Br Med J. 1996;312(7030):572

37. Morley JE, Kraenzle D. Causes of weight loss in a community nursing home. J Am Geriatr Soc. 1994;42(6):583-5.

38. Alibhai SM, Greenwood C, Payette H. An approach to the management of unintentional weight loss in elderly people. Can Med Assoc J. 2005;172(6):773-80.

39. Galanos AN, Pieper CF, Cornoni-Huntley JC, Bales CW, Fillenbaum GG. Nutrition and function: is there a relationship between body mass index and the functional capabilities of community-dwelling elderly? J Am Geriatr Soc. 1994;42(4):368-73.

40. Fried L, Walston J. Frailty and failure to thrive. En: Hazzard W, Blass J, Ettinger W, Halter J, Ouslander J, eds. Principles of geriatric medicine and gerontology. 5th ed. New York: McGraw-Hill; 2003. Pp. 1487-502.

41. Rao R. Cerebrovascular disease and late life depression: an age old association revisited. Int J Geriatr Psychiatry. 2000;15(5):419-33.
42. Firbank MJ, Lloyd AJ, Ferrier N, O'Brien JT. A volumetric study of MRI signal hyperintensities in late-life depression. Am J Geriatr Psychiatry. 2004;12(6):606-12.

43. Husain MM, McDonald WM, Doraiswamy PM, Figiel GS, Na C, Escalona PR, et al. A magnetic resonance imaging study of putamen nuclei in major depression. Psychiatry Res. 1991;40(2):95-9.

44. Van Iersel MB, Haitsma A, Olde Rikkert MG, Benraad CE. Quantitative gait analysis to detect gait disorders in geriatric patients with depression. J Am Geriatr Soc. 2005;53(8): 1441-2.

45. Reding M, Haycox J, Blass J. Depression in patients referred to a dementia clinic. A threeyear prospective study. Arch Neurol. 1985; 42(9):894-6.

46. Alexopoulos GS, Meyers BS, Young RC, Mattis S, Kakuma T. The course of geriatric depression with "reversible dementia": a controlled study. Am J Psychiatry. 1993;150(11): 1693-9.

47. Ringback Weitoft G, Rosen M. Is perceived nervousness and anxiety a predictor of premature mortality and severe morbidity? A longitudinal follow up of the Swedish Survey of Living Conditions. J Epidemiol Community Health. 2005;59(9):794-8.

48. Blazer DG. Depression in late life: review and commentary. J Gerontol A Biol Sci Med Sci. 2003;58(3):249-65.

49. Little JW. Dental implications of mood disorders. Gen Dent. 2004;52(5):442-50.

50. Weyant RJ, Pandav RS, Plowman JL, Ganguli $\mathrm{M}$. Medical and cognitive correlates of denture wearing in older community-dwelling adults. J Am Geriatr Soc. 2004;52(4):596-600.

51. Kiosses DN, Alexopoulos GS. IADL functions, cognitive deficits, and severity of depression: a preliminary study. Am J Geriatr Psychiatry. 2005;13(3):244-9.

52. Klesges LM, Pahor M, Shorr RI, Wan JY, Williamson JD, Guralnik JM. Financial difficulty in acquiring food among elderly disabled women: results from the Women's Health and Aging Study. Am J Public Health. 2001;91(1):68-75.

53. Penninx BW, Leveille S, Ferrucci L, van Eijk JT, Guralnik JM. Exploring the effect of depression on physical disability: longitudinal evidence from the established populations for epidemiologic studies of the elderly. Am J Public Health. 1999;89(9):1346-52.

54. Stewart AL. The reliability and validity of self-reported weight and height. J Chronic Dis. 1982;35(4):295-309.

55. Ávila-Funes JA, Gutiérrez-Robledo LM, Ponce de León Rosales S. Validity of height and weight self-report in Mexican adults: results from the national health and aging study. J Nutr Health Aging. 2004;8(5):355-61.

Manuscrito recibido el 14 de septiembre de 2005. Aceptado para publicación, tras revisión, el 13 de marzo de 2006. 
ABSTRACT Objective. To determine the factors that are commonly associated with both the dietary habits of older adults living in the community and depressive symptoms in this group. Methods. Secondary analysis of data on Mexico City obtained by means of the multi-

Relationship between determining factors for depressive symptoms and for dietary habits in older adults in Mexico

center study on Health, Well-being, and Aging (Salud, Bienestar y Envejecimiento, SABE) that was carried out in 1999 and 2000. The following variables were examined: presence or absence of depressive symptoms, according to Yesavage's Geriatric Depression Scale (GDS); mental status, as reflected by the score obtained on a modified, shortened version of Folstein's Mini-mental State Examination (MMSE); and functional capacity, as measured by Katz' Index of Independence in Activities of Daily Living. Those whose score on the shortened MMSE was 12 points or less were asked to respond to the Pfeffer Functional Activities Questionnaire, which was used to assess their ability to perform the basic activities of daily living. Information was obtained through self-report on the material, physical, psychological, and social aspects of purchasing, preparing, and consuming food products and of oral health status during the most recent 12-month period. Variables that were significantly associated with the results obtained on the GDS were included in a multivariate regression analysis; several statistical models were created, and variables that were shown to be statistically significant in the stepwise multivariate linear regression were used to determine the best-fitting explanatory model for the results obtained on the GDS.

Results. The average age of study participants was $64.4 \pm 8.6$ years, and the prevalence of depressive symptoms was $66 \%$. The score obtained on the GDS showed a significant association with the presence of arterial hypertension $(P<0.01)$, but not with the self-reported presence of diabetes, neoplasia, stroke, lung disease or heart disease. However, the use of dental prostheses $(P<0.01)$, urinary incontinence $(P<0.01)$, and falls $(P<0.01)$ were significantly associated with the results on the GDS. The intake of milk products, meat, fish, fowl, fruit, and vegetables was significantly lower in the group that had depressive symptoms. An inverse correlation was detected between the score obtained on the GDS on the one hand, and the number of complete meals consumed during the day $(P<0.01)$ and total fluid intake $(P<0.01)$ on the other. The determining factors that were most closely associated with these results were, in addition to the presence of arterial hypertension, the presence of cognitive impairment $(P<0.01)$, difficulty performing the basic activities of daily living $(P=0.03)$ and the instrumental activities of daily living $(P<0.01)$, poor mobility $(P<0.01)$, difficulty using the telephone $(P<0.01)$, and the self-perception of having poor memory $(P<0.01)$, of having insufficient resources with which to live $(P<0.01)$, and of having poor oral health $(P<0.01)$. These variables explained $31 \%$ of the variance seen in the $R^{2}$ values linked to the SDG variables that were incorporated into the final explanatory model. Conclusion. A number of determining factors for depressive symptoms and the results obtained on the GDS resemble the factors that determine poor dietary habits among older adults. The potential existence of common causative mechanisms calls attention to the need for designing interventions aimed at preventing both types of problems and their negative consequences. These results confirm the need to take dietary habits and other parameters into account when studying depression in older adults.

Key words Depression, malnutrition, aged, feeding, Mexico. 